\title{
Programmed Cell-Death Mechanism Analysis Using Same-Cell, Multimode DNA and Proteoform Electrophoresis
}

Ana E. Gomez Martinez ${ }^{1,2}$ and Amy E. Herr*1,2,3.

${ }^{1}$ Department of Bioengineering, University of California Berkeley, Berkeley, California, 94720, USA. ${ }^{2}$ The

University of California Berkeley and University of California San Francisco Graduate Program in Bioengineering, Berkeley, California, 94720, USA. ${ }^{3}$ Chan Zuckerberg Biohub, San Francisco, California, 94158, USA.

*aeh@berkeley.edu

\section{Supporting Information}

Mycoplasma testing and Cell Line Short Tandem Repeat (STR) Report are from 2019. Experiments were performed between July 2019 and October 2020. 


\title{
Berkeley Molecular \& Cell Biology
}

\author{
University of California, Berkeley \\ Cell Culture Facility
}

Certificate of Analysis

Cell Line Short Tandem Repeat (STR) Report

Sample name: SKBR3

Date received: 10/30/19

Client: Alisha Geldert-UCB

\begin{tabular}{|l|l|}
\hline LOCUS & ALLELE SIZE \\
\hline THO1 & 8,9 \\
\hline D5S818 & 9,12 \\
\hline D13S317 & 11,12 \\
\hline D7S820 & 9,12 \\
\hline D16S539 & 9 \\
\hline CSF1PO & 12 \\
\hline AMEL & $X$ \\
\hline VWA & 17 \\
\hline TPOX & 8,11 \\
\hline
\end{tabular}

Results indicate the allele(s) detected at each locus tested. Each allele represents the number of short tandem repeats present at that locus. Generally, a DNA profile uniquely identifies an individual cell line. However, some cell lines may exhibit genomic instability over time leading to slight changes in the DNA profile.

\begin{tabular}{|l|l|}
\hline IDENTIFIED CELL TYPE(S) & PERCENT MATCH \\
\hline SK-BR-3 & $100 \%$ \\
\hline
\end{tabular}

Based on STR analysis, the results indicate a high probability $(80 \%$ or higher) match with the cell line \#1 listed. Multiple cell lines with $\geq 80 \%$ match are considered to be derived from common ancestry. Electropherograms are available upon request.

Disclaimer: While every reasonable effort has been made to assure the accuracy of these data, no warranty, express or implied, is made by this facility.

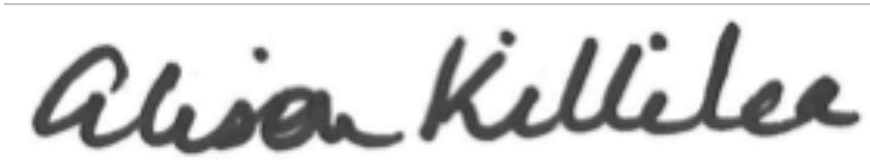

Alison N. Killilea, Ph.D. | Bioscience Facility Manager 


\section{Molecular \& Cell Biology}

University of California, Berkeley

Cell Culture Facility

Certificate of Analysis

Mycoplasma Testing

Sample names: BT474, SK-BR-3, MCF-7 naive, MDA-MB-231, HEK 293, CHO-p185, CHO-p110, CHO-p95, U-251 + TurboGFP, MDA-MB-231 GFP Actin, MDA-MB-231 sh scramble GFP Actin, MDA-MB-231 GFP Actin+HSF1 OE, MDA-MB-231 GFP Actin +RFP lenti, MDA-MB-231 GFP Actin+sh HSF1B, MDA-MB-231 GFP control, BJ

fibroblasts, U251 naïve, HeLa

Date tested: $10 / 31 / 19$

Client: Alisha Geldert

Mycoplasma test: NEGATIVE 10/31/19: Cells were fixed with methanol and stained with Hoechst nuclear stain to visualize nuclei. Small nuclei present in the cellular membrane indicate mycoplasma infection.

Disclaimer: While every reasonable effort has been made to assure the accuracy of these data, no warranty, express or implied, is made by this facility.

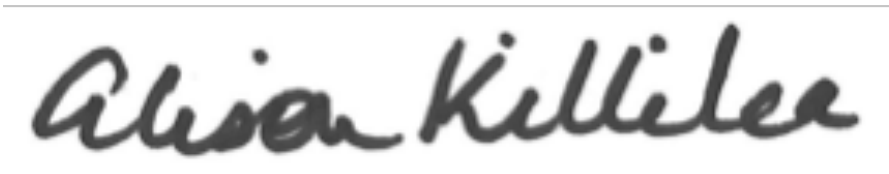

Alison N. Killilea, Ph.D. | Bioscience Facility Manager 